\title{
The role of TNF- $\alpha$ in regulating ketamine-induced hippocampal neurotoxicity
}

\author{
Xiaozhu Zheng, Jiali Zhou, Yanfei Xia \\ Department of Anesthesia, Zhejiang Hospital, Hangzhou, Zhejiang, China \\ Submitted: 31 December 2013 \\ Accepted: 9 March 2014 \\ Arch Med Sci 2015; 11, 6: 1296-1302 \\ DOI: 10.5114 /aoms.2015.56355 \\ Copyright @ 2015 Termedia \& Banach \\ Corresponding author: \\ Jiali Zhou MD \\ Department of Anesthesia \\ Zhejiang Hospital \\ 12 Lingyin Rd \\ Hangzhou, Zhejiang \\ 310003 China \\ Phone: +86-13588178819 \\ E-mail: jiali.zhou@aol.com
}

\begin{abstract}
Introduction: Ketamine is commonly used in pediatric anesthesia but recent studies have shown that it could induce neurotoxicity in the developing brain. The inflammatory cytokine, tumor necrosis factor $\alpha$ (TNF- $\alpha$ ) is involved in the pathogenesis of various types of neurodegenerations. In the present study, we examined whether TNF- $\alpha$ may regulate ketamine-induced neurotoxicity in the hippocampus of neonatal mouse.

Material and methods: The in vitro organotypic culture of hippocampal slices was used to investigate the gain-of-function and loss-of-function effect of TNF- $\alpha$ modulation on ketamine-induced hippocampal neurotoxicity. Also, western blotting analysis was used to examine the relative pathways associated with TNF- $\alpha$ modulation. In the in vivo Morris water maze test, TNF- $\alpha$ was genetically silenced to see if memory function was improved after anesthesia-induced memory impairment.

Results: In in vitro experiments, adding TNF- $\alpha$ enhanced (112.99 $\pm 5.4 \%$, $p=0.015)$, whereas knocking down TNF- $\alpha$ ameliorated (46.8 $\pm 11.6 \%$, $p=0.003)$ ketamine-induced apoptosis in hippocampal CA1 neurons in the organotypic culture. Western blotting showed that addition of TNF- $\alpha$ reduced $(67.1 \pm 3.7 \%, p=0.022)$, whereas downregulation of TNF- $\alpha$ increased (126.87 $\pm 8.5 \%, p=0.004)$ the phosphorylation of PKC-ERK pathway in ketamine-treated hippocampus. In in vivo experiments, genetically silencing TNF- $\alpha$ markedly improved the ketamine-induced memory impairment through Morris water maze test.

Conclusions: Our results clearly demonstrated a protective mechanism of down-regulating TNF in ketamine-induced hippocampal neurotoxicity. This study may present a new target for pharmacological intervention to prevent anesthesia-related neurodegeneration in brain.
\end{abstract}

Key words: tumor necrosis factor- $\alpha$, ketamine, hippocampus, neurotoxicity, anesthesia.

\section{Introduction}

Ketamine is a widely used pediatric anesthetic mainly working through the blockage of N-methyl-D-aspartate (NMDA)-type glutamate receptors [1, 2]. It was discovered decades ago that excessive expose of ketamine or other NMDA antagonists caused apoptosis in the neonatal rat brain [3]. Since then, an increasing number of studies have shown that ketamine-induced neurotoxicity may affect the development of young brains in various species, including humans [4-7]. Most recently, studies demonstrated that repeated or high dosages of ketamine ad- 
ministration could induce apoptosis in the neonatal hippocampus, leading to memory impairment [8-11]. However, the exact mechanisms of anesthesia-induced hippocampal neurodegeneration are largely unknown.

Tumor necrosis factor- $\alpha$ (TNF- $\alpha$ ), a prototypical inflammatory cytokine, is widely involved in various biological or pathological processes, including inflammation, the immune response, apoptosis and necrosis, and neurodegenerative diseases [12-16]. In the central nervous system, various downstream targets, sometimes paradoxical ones, could be activated by TNF- $\alpha$, which may exert contradictory effects on neurons, including both neuroprotective and neurotoxic ones, depending on the sites or the pathologic conditions of the brain [17-19]. Interestingly, in the hippocampus, TNF- $\alpha$ was shown to be able to either ameliorate or augment neuronal apoptosis after ischemic injury [17, 20], suggesting an active yet complex role of TNF- $\alpha$ in modulating neuronal activities in the hippocampus. In the present study, we intended to examine the role of TNF- $\alpha$ in regulating ketamine-induced neurotoxicity in the neonatal mouse hippocampus both in vitro and in vivo. Our results would undoubtedly gain invaluable information on the underlying mechanisms of TNF pathways in regulating anesthesia-induced hippocampal neurodegeneration.

\section{Material and methods}

\section{Organotypic hippocampal-slice cultures}

Wilde type C57BL/6J mice, postnatal 7 to 10 days old (P7 - P10) were purchased from The Jackson Laboratory (Bar Harbor, ME, USA). The method of obtaining and culturing organotypic hippocampal-slice cultures was described in previous studies [21, 22]. Briefly, neonatal mice were anesthetized on an ice block and sacrificed by decapitation. The hippocampi were retrieved and transverse sections $(350 \mu \mathrm{m})$ were cut with a customized tissue chopper and quickly incubated into a petri-dish containing ice-cold Gey's balanced salt solution including glucose and 1.5\% Fungizone (Invitrogen, USA). The hippocampal slices were then maintained in an incubator at $37^{\circ} \mathrm{C}$ with $5 \% \mathrm{CO}_{2}$, with the culture medium of $50 \%$ MEM, 25\% Hanks' balanced salt solution, 25\% heat inactivated bovine serum, $5 \mathrm{mg} / \mathrm{ml}$ glucose, $1 \mathrm{mmol} / \mathrm{l}$ glutamine, and 1.5\% Fungizone.

\section{In vitro neurotoxicity treatment}

Ketamine was purchased from Jiangsu Hengrui Medicine Co., Ltd (Jiangsu, China). TNF- $\alpha$ was purchased from Sigma (St. Louis, MO, USA). TNF- $\alpha$ SiRNA was purchased from IDT Inc. (Coralville, IA, USA). To induce neurotoxicity, the hippocampal slices were treated with ketamine $(0.5 \mathrm{mM})$ for $4 \mathrm{~h}$ af- ter overnight culture, followed by $3 \times 10 \mathrm{~min}$ wash at $37^{\circ} \mathrm{C}$ with $5 \% \mathrm{CO}_{2}$. After that, to examine the effect of TNF- $\alpha$ through a gain-of-function paradigm, TNF- $\alpha(50 \mathrm{ng} / \mathrm{ml})$ was added into the organotypic hippocampal culture for $24 \mathrm{~h}$. For the loss-of-function paradigm, TNF- $\alpha$ SiRNA (100 nM) was instead introduced into the organotypic hippocampal culture through a gene silencer transfection reagent according to the manufacturer's protocol (GenLentis, CA, USA). The cultures were then maintained for another $24 \mathrm{~h}$ before immunohistochemistry and western blotting analysis. The efficacy of TNF- $\alpha$ SiRNA was confirmed by western blotting (Figure $1 \mathrm{~A}$ ).

\section{In vitro TUNEL staining for cell death detection}

The apoptosis of hippocampal CA1 neurons was assessed with immunohistochemical labeling of terminal deoxynucleotidyl transferase-mediated biotinylated UTP nick end labeling (TUNEL) according to the manufacturer's protocol (in situ cell death detection kit, Roche, USA). Cells with DNA fragmentation were determined by the terminal deoxynucleotidyl transferase incorporation of TRITC-12-dUTP into DNA. The average number of TUNEL-positive cells in the CA1 region (per $0.01 \mathrm{~mm}^{2}$ ) for each experimental condition was assessed, and then normalized to the value under control conditions.

\section{Western blot analysis}

Hippocampal slices were homogenized with Tris- $\mathrm{HCl}$ buffer including (in $\mathrm{mM}$ ) 0.5 EDTA and 250 sucrose and centrifuged at $15,000 \mathrm{rpm}$ for $25 \mathrm{~min}$ at $4^{\circ} \mathrm{C}$. The protein was extracted from supernatant by SDS-PAGE on $10 \%$ acrylamide gels and then transferred onto polyvinylidene fluoride (PVDF) membranes and blocked with 5\% nonfat dry milk in a Tris-Buffered Saline Tween-20 (TBST) buffer (50 Tris- $\mathrm{HCl}, 150 \mathrm{mmol} / \mathrm{l} \mathrm{NaCl}, 0.1 \%$ Tween, $\mathrm{pH}$ 7.4) for $1 \mathrm{~h}$ at RT. For primary antibodies, $\mathrm{p}$-PKC was purchased from Abcam (Cambridge, UK), p-ERK and TNF- $\alpha$ from Santa Cruz (CA, USA). The peroxidase-labeled affinity purified secondary antibody rabbit IgG and mouse IgG were purchased from Sigma (St. Louis, USA). After overnight incubation of primary antibody followed by 1 -hour incubation of secondary antibody, blots were detected by X-ray film and quantified densitometric measurements through three independent repeats.

\section{In vivo neurotoxicity treatment and surgery}

Wilde type P14 C57BL/6J mice were intraperitoneally injected with $75 \mathrm{mg} / \mathrm{kg}$ ketamine once a day for 3 consecutive days to induce anesthesia-related memory loss according to previous studies [8, $9,11]$. Twenty-four hours after the last ketamine 
A

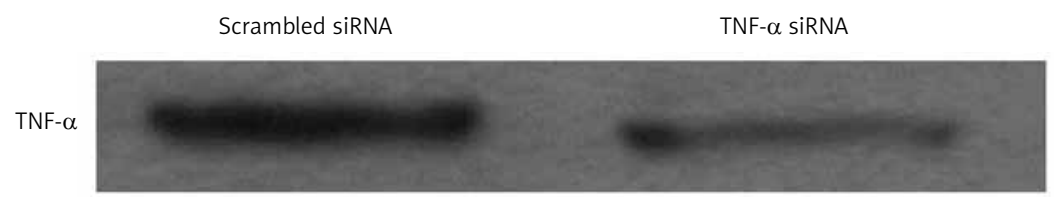

$\beta$-Actin

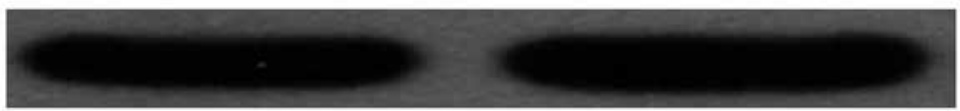

B
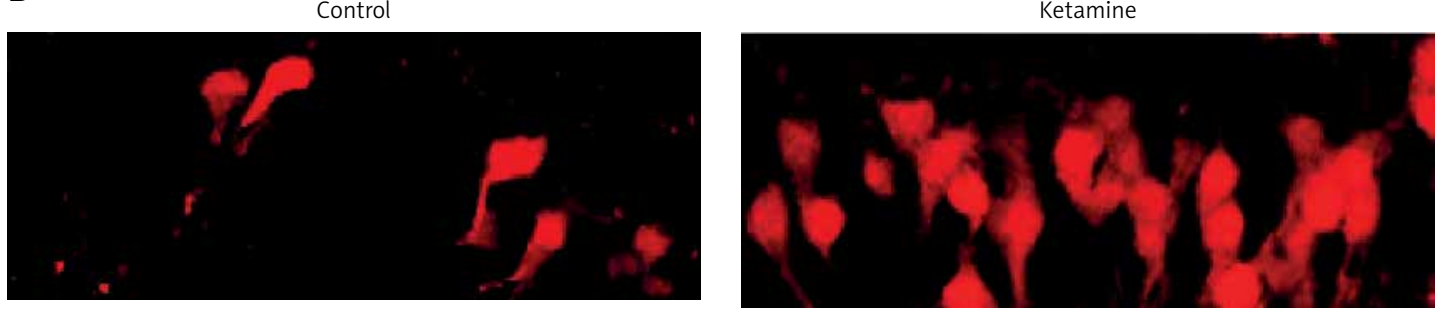

Ketamine + TNF- $\alpha$

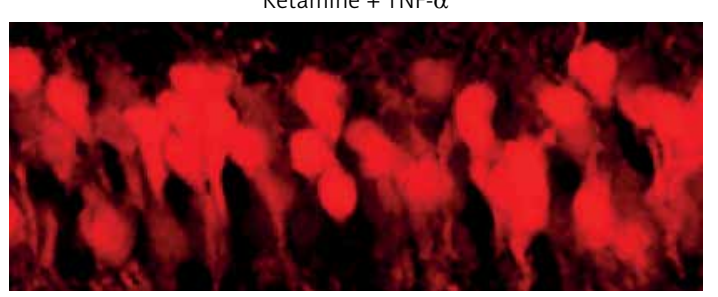

Ketamine + TNF- $\alpha$ siRNA
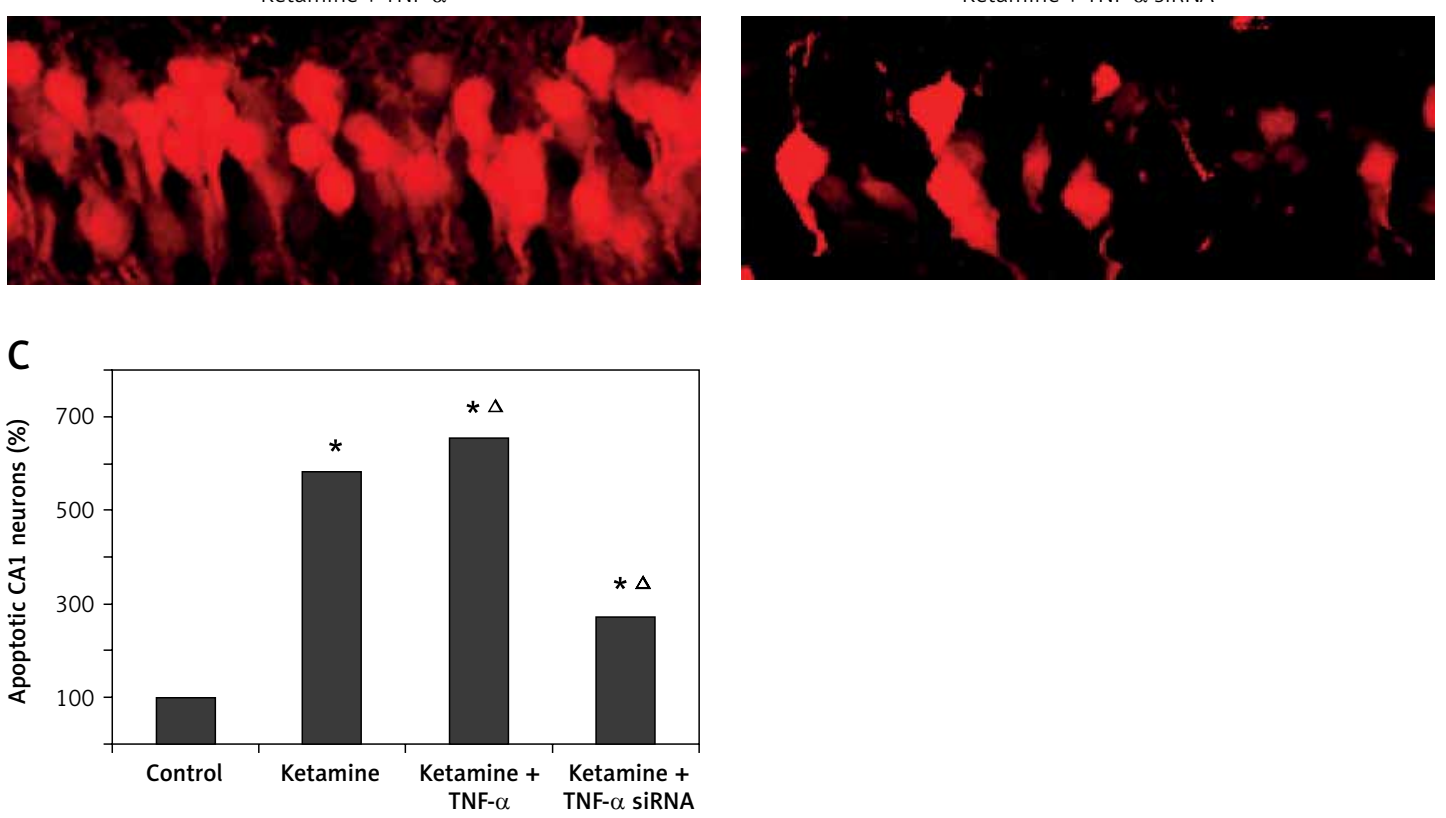

Figure 1. TNF- $\alpha$ regulated ketamine-induced neurotoxicity in vitro. A - The hippocampal cultures were cultured with TNF- $\alpha$ siRNA $(100 \mu \mathrm{M})$ and nonspecific scrambled siRNA $(100 \mu \mathrm{M})$ for $24 \mathrm{~h}$. The efficacy of TNF- $\alpha$ siRNA was then verified by western blotting. B - Representative images of TUNEL-positive CA1 neurons of organotypic hippocampal slice cultures that were treated with normal medium (control), $4 \mathrm{~h}$ of $0.5 \mathrm{mM}$ ketamine (ketamine) only, $4 \mathrm{~h}$ of $0.5 \mathrm{mM}$ ketamine plus $50 \mathrm{ng} / \mathrm{ml}$ TNF- $\alpha$ treatment (ketamine + TNF- $\alpha$ ), or $4 \mathrm{~h}$ of $0.5 \mathrm{mM}$ ketamine plus 100 nM TNF- $\alpha$ SiRNA treatment (ketamine + TNF- $\alpha$ siRNA). C - Quantitative measurements showed that adding TNF- $\alpha$ significantly increased the number of TUNEL-positive CA1 neurons whereas silencing TNF- $\alpha$ significantly reduced the number of TUNEL-positive CA1 neurons. ${ }^{\star} P<0.05$, as compared to control, ${ }^{\Delta} p<0.05$, as compared to ketamine treatment $(n=3)$

administration, mice were quickly anesthetized by isoflurane and the right side of the cranium was exposed. A hole was drilled over the hippocampal injection site and the injection was conducted by a Hamilton syringe at the following coordinates, calculated from the bregma and skull surface: anteroposterior $-2.0 \mathrm{~mm}$, lateral $+1.0 \mathrm{~mm}$, and vertical $-1.5 \mathrm{~mm}$ [23]. The injections were $1 \mu \mathrm{l}$ total volume of either TNF- $\alpha$ siRNA (20 mM) or scram- bled siRNA (20 mM, control). The incision was then cleaned and closed. The mice were awakened in $15 \mathrm{~min}$ and returned to their mothers.

\section{Morris water maze test}

The Morris water maze test was performed at 8 weeks of age to examine the memory deficits in the ketamine-induced neurotoxic mice. The Morris water maze was filled with warm water at $22^{\circ} \mathrm{C}$ and 
the platform was submerged $2 \mathrm{~cm}$ below the water. Visual cues of black-and-white paints and a heating lamp were positioned on the walls. The mice were given a series of acquisition training sessions of four trials per day for 4 days before examination on the $5^{\text {th }}$ day. They were allowed to swim to locate the platform in $120 \mathrm{~s}$ and then stay there for $30 \mathrm{~s}$. If mice did not locate the platform within $120 \mathrm{~s}$, they were manually guided to swim to it and stay there for $30 \mathrm{~s}$. A computerized auto-tracking digital camera system was used to record and analyze the time for mice to reach the platform (latency) and the length of the swim route (distance). Finally, at the end of the training sessions, the platform was removed and the mice were allowed to swim freely for $2 \mathrm{~min}$, examined in a spatial probe trial in which the platform was removed, and they were allowed to swim freely for an additional $2 \mathrm{~min}$.

\section{Statistical analysis}

Data analysis was conducted using SAS software (version 11). The data are presented as mean \pm SD. The comparison of data was conducted by two-tail, unpaired Student's t-test. A $p$ value $<0.05$ was considered significant.

\section{Results}

\section{Tumor necrosis factor- $\alpha$ augmented ketamine-induced hippocampal neurotoxicity in vitro}

We used an organotypic hippocampal-slice culture model to introduce ketamine-related neurotoxicity in CA1 neurons in neonatal mice [10]. The in vitro hippocampal slices were initially cultured with or without $0.5 \mathrm{mM}$ ketamine for $4 \mathrm{~h}$, followed by wash and overnight incubation. The result showed that ketamine treatment induced significant apoptosis, based on TUNEL staining, in CA1 neurons (Figure $1 \mathrm{~B}$, top panel; Figure $1 \mathrm{C}$ ).

We then examined whether upregulating TNF- $\alpha$ might affect ketamine-induced hippocampal apoptosis. On the second day of culture, for the slices that were previously treated with ketamine, $50 \mathrm{ng} / \mathrm{ml} \mathrm{TNF-} \alpha$ was added to the culture for another $24 \mathrm{~h}$ before immunohistochemistry of TUNEL staining. Our results demonstrated that more CA1 neurons underwent apoptosis with the addition of TNF- $\alpha$ after ketamine treatment (Figure $1 \mathrm{~B}$, bottom panel, left), and the difference was significant (Figure $1 \mathrm{C}$ ).

\section{Knocking down tumor necrosis \\ factor- $\alpha$ reduced neurotoxicity in ketamine-treated hippocampus in vitro}

Since up-regulating TNF- $\alpha$ augmented the neurotoxic effect in the ketamine-treated hippo- campus, we then asked whether down-regulating TNF- $\alpha$ would exert the opposite effect. After ketamine treatment, some of the cultured hippocampal slices were further treated with TNF- $\alpha$ SiRNA (100 nM) for $24 \mathrm{~h}$ to genetically knock down TNF- $\alpha$ in the hippocampus. With examination of TUNEL staining, we found that down-regulating TNF- $\alpha$ reduced the number of apoptotic neurons in the hippocampus (Figure $1 \mathrm{~B}$, bottom panel, right), and the difference was also significant (Figure $1 \mathrm{C}$ ).

\section{Tumor necrosis factor- $\alpha$ regulated \\ ketamine-induced hippocampal \\ neurotoxicity through the PKC-ERK pathway in vitro}

Previous studied demonstrated that the PKCERK pathway was downregulated during the process of ketamine-mediated neuronal death in the hippocampus $[9,11]$. As we discovered that TNF- $\alpha$ played an important role in regulating ketamine-induced neurotoxicity in the hippocampus, we turned to biochemistry to investigate whether TNF- $\alpha$ modulated hippocampal apoptosis through the PKC-ERK signaling pathway. Our western blotting assay showed that adding a compound of TNF- $\alpha$ to the ketamine-treated hippocampal culture further reduced the protein expression of phosphorylated ERK ( $p$-ERK) and phosphorylated PKC ( $p-P K C)$, whereas silencing TNF- $\alpha$ upregulated the expression of $p$-ERK and $p$-PKC (Figure $2 A$ ). Quantitative analysis showed that the attenuating and augmenting effects of TNF- $\alpha$ on PKC pathway related proteins were significant (Figure $2 \mathrm{~B}$ ).

\section{Tumor necrosis factor- $\alpha$ improved ketamine-induced memory impairment in vivo}

Finally, we asked whether the modulatory effects of TNF- $\alpha$ on ketamine-induced neurodegeneration in vitro could be recapitulated in the in vivo experiments. Young mice at the age of postnatal 14 days were intraperitoneally injected with a daily dose of $75 \mathrm{mg} / \mathrm{kg}$ ketamine for 3 days to induce anesthesia-specific memory impairment [9]. On the $4^{\text {th }}$ day, the mice were introduced with cortical injection of either non-specific control scrambled SiRNA $(20 \mathrm{mM})$ or TNF- $\alpha$ SiRNA $(20 \mathrm{mM})$ in the hippocampus. At 2 months, mice were examined with the Morris water maze test to examine the effect of TNF- $\alpha$ on memory. The test showed that after knocking down TNF- $\alpha$, mice spent significantly less time (Figure $3 \mathrm{~A}$ ) and shorter distances (Figure $3 \mathrm{~B}$ ) in finding the platform when compared with mice without TNF- $\alpha$ silencing. Thus, our results strongly suggested that down-regulating TNF- $\alpha$ in the hippocampus markedly ameliorated the memory impairment induced by ketamine. 

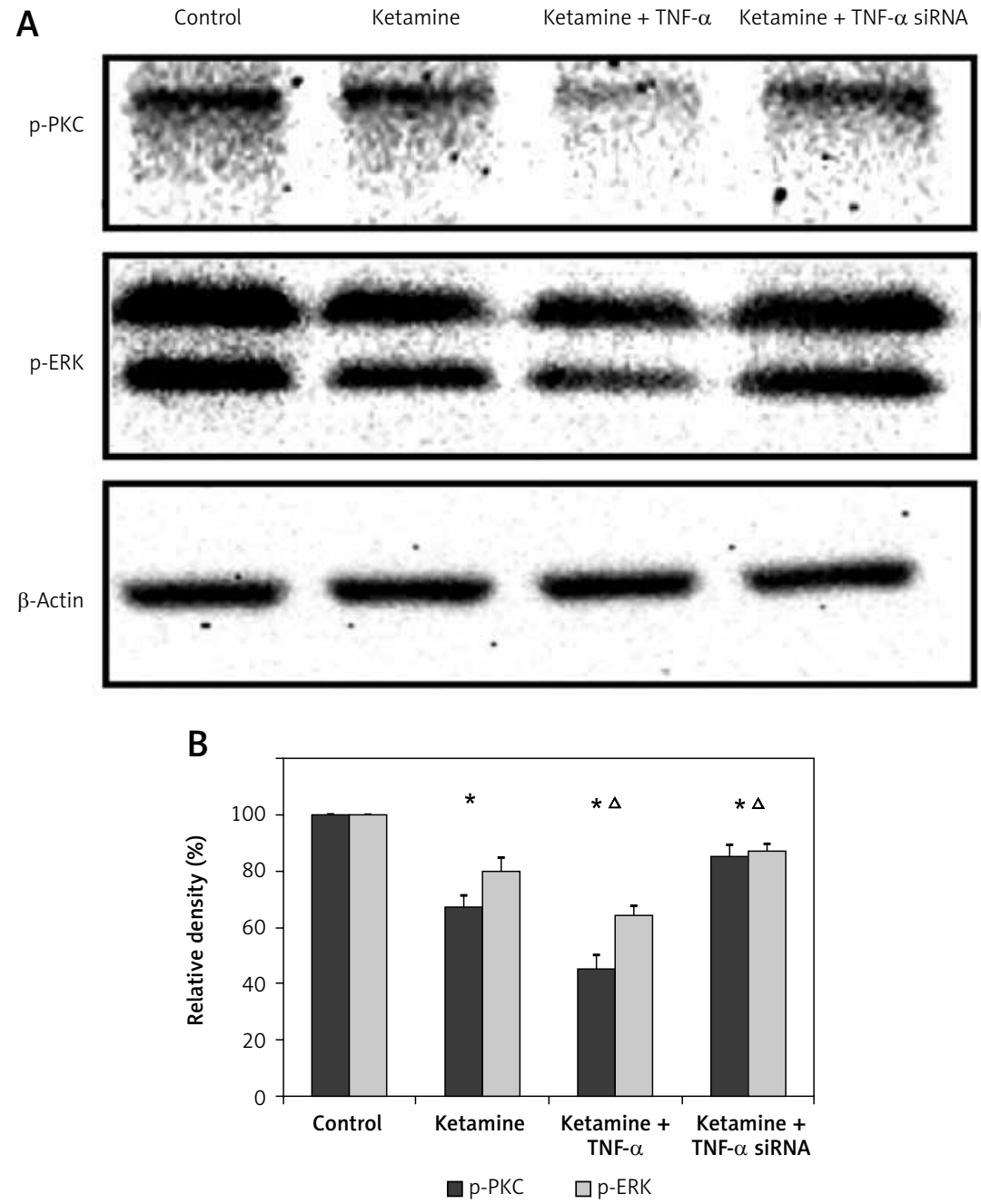

Figure 2. TNF- $\alpha$ regulated PKC-ERK signaling pathway during ketamine-induced neurotoxicity in vitro. A - Western blotting analysis of the protein expression levels of phosphorylated PKC (p-PKC) and phosphorylated ERK ( $p$-ERK) for the organotypic hippocampal slice cultures that were treated with control medium, ketamine only, ketamine + TNF- $\alpha$, or ketamine + TNF- $\alpha$ siRNA. B - Semi-quantitative measurements of the protein densities when hippocampal cultures were treated with control medium, ketamine only, ketamine + TNF- $\alpha$, or ketamine + TNF- $\alpha$ siRNA. ${ }^{*} P<0.05$, as compared to control, ${ }^{\Delta} p<0.05$, as compared to ketamine treatment $(n=3)$

A

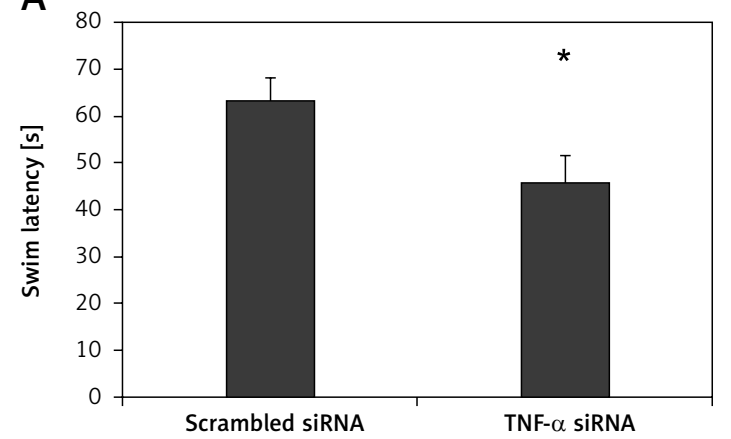

B

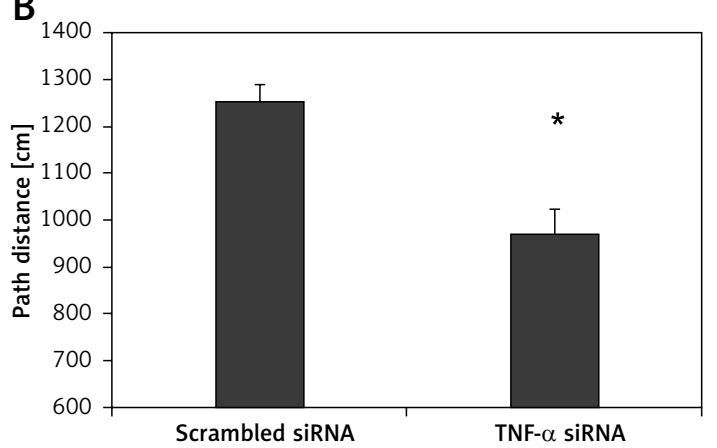

Figure 3. TNF- $\alpha$ reduced ketamine-induced memory loss in Morris water maze test. Neonatal mice (P14) were systemically injected with ketamine, followed by knocking down TNF- $\alpha$ siRNA injection (20 mM) in the hippocampus. The control mice were injected with non-specific scrambled SiRNA ( $20 \mathrm{mM})$. At the age of 2 months, the Morris water maze test was performed and the data of swim latency $(\mathbf{A})$ and swimming path distance (B) were compared between mice injected with control siRNA and TNF- $\alpha$ siRNA. ${ }^{*} p<0.05(n=7)$ 


\section{Discussion}

As an NMDA receptor antagonist, ketamine is ideal to be used in neonatal anesthesia. However, its neurotoxic side-effects have attracted more and more attention both academically and clinically, as effective therapeutic interventions are mainly unavailable [7, 24]. In the present study, we demonstrated that, in both in vitro and in vivo experiments, blocking TNF- $\alpha$ could attenuate ketamine-induced neurotoxicity in the neonatal mouse hippocampus, suggesting that TNF- $\alpha$ played an important role in modulating anesthesia-related neurodegeneration in the hippocampus.

While the exact mechanisms of ketamine-induced hippocampal neurotoxicity remain elusive, a recent study demonstrated that blockage of the NMDA receptor by ketamine attenuated spontaneous $\mathrm{Ca}^{2+}$-dependent membrane potential oscillations in hippocampal neurons to induce apoptosis [10]. This interesting finding strongly suggested that ionic homeostasis of hippocampal neurons, especially through the modulation of glutamate receptors, may decisively affect the pathologic condition of apoptosis in the hippocampus. Interestingly, the family of tumor necrosis factors, especially TNF- $\alpha$, has been shown to induce overexpression of glutamate AMPA receptors in the hippocampal neurons to cause excitotoxicity $[17,25]$. Thus, it is very likely that the combination of ketamine, through modulation of NMDA glutamate receptors, and TNF- $\alpha$, through modulation of AMPA glutamate receptors, exerted synergistic effects to unbalance the calcium or other ionic homeostasis and induce apoptosis in the hippocampal neurons. However, future studies would be needed to further elucidate how exactly the cross-talk between these two seemingly different mechanisms, neuronal inhibition by blockage of NMDA, and neuronal excitation by overexpressing AMPA, would yield an additive cellular effect on neurodegeneration.

In conclusion, our study clearly demonstrated a protective mechanism of down-regulating TNF- $\alpha$ in ketamine-induced hippocampal neurotoxicity. The experimental results are invaluable for the understanding of the TNF signaling pathway in regulating anesthesia-related neurodegeneration in the hippocampus, as well as finding a new pharmacological target to treat anesthesia-induced neurotoxicity in developing brains in children.

\section{Conflict of interest}

The authors declare no conflict of interest.

\section{References}

1. Ben-David B. NMDA receptor blockade: from the laboratory to clinical application. Anesth Analg 2000; 91: 1042-3.
2. Kohrs R, Durieux ME. Ketamine: teaching an old drug new tricks. Anesth Analg 1998; 87: 1186-93.

3. Ikonomidou C, Bosch F, Miksa M, et al. Blockade of NMDA receptors and apoptotic neurodegeneration in the developing brain. Science 1999; 283: 70-4.

4. McGowan FX Jr, Davis PJ. Anesthetic-related neurotoxicity in the developing infant: of mice, rats, monkeys and, possibly, humans. Anesth Analg 2008; 106: 1599-602,

5. Stratmann G. Neurotoxicity of anesthetic drugs in the developing brain. Anesth Analg 2011; 113: 1170-9.

6. Brambrink AM, Orfanakis A, Kirsch JR. Anesthetic neurotoxicity. Anesthesiol Clin 2012; 30: 207-28.

7. Wang C, Liu F, Patterson TA, Paule MG, Slikker W Jr. Preclinical assessment of ketamine. CNS Neurosci Ther 2013; 19: 448-53.

8. Hayashi H, Dikkes P, Soriano SG. Repeated administration of ketamine may lead to neuronal degeneration in the developing rat brain. Paediatr Anaesth 2002; 12: 770-4.

9. Huang L, Liu Y, Jin W, Ji X, Dong Z. Ketamine potentiates hippocampal neurodegeneration and persistent learning and memory impairment through the PKCgamma-ERK signaling pathway in the developing brain. Brain Res 2012; 1476: 164-71.

10. Huang L, Liu Y, Zhang P, et al. In vitro dose-dependent inhibition of the intracellular spontaneous calcium oscillations in developing hippocampal neurons by ketamine. PLoS One 2013; 8: e59804.

11. Peng S, Zhang Y, Zhang J, Wang H, Ren B. Effect of ketamine on ERK expression in hippocampal neural cell and the ability of learning behavior in minor rats. Mol Biol Rep 2010; 37: 3137-42.

12. McCoy MK, Ruhn KA, Blesch A, Tansey MG. TNF: a key neuroinflammatory mediator of neurotoxicity and neurodegeneration in models of Parkinson's disease. In: Advances in TNF family research. Springer, 2011; 539-40.

13. Peyrin-Biroulet L. Anti-TNF therapy in inflammatory bowel diseases: a huge review. Minerva Gastroenterol Dietol 2010; 56: 233-43.

14. Wang CY, Mayo MW, Baldwin AS. TNF-and cancer therapy-induced apoptosis: potentiation by inhibition of NF-kappaB. Science 1996; 274: 784-7.

15. Baeuerle PA, Henkel T. Function and activation of NF-kappaB in the immune system. Ann Rev Immunol 1994; 12: 141-79.

16. Paluch-Oles J, Magrys A, Koziol-Montewka M, Koszarny A, Majdan M. Identification of latent tuberculosis infection in rheumatic patients under consideration for treatment with anti-TNF-alpha agents. Arch Med Sci 2013; 9: 112-7.

17. Leonoudakis D, Braithwaite SP, Beattie MS, Beattie EC. TNFalpha-induced AMPA-receptor trafficking in CNS neurons; relevance to excitotoxicity? Neuron Glia Biol 2004; 1: 263-73.

18. Perry SW, Dewhurst S, Bellizzi MJ, Gelbard HA. Tumor necrosis factor-alpha in normal and diseased brain: Conflicting effects via intraneuronal receptor crosstalk? J Neurovirol 2002; 8: 611-24.

19. McCoy MK, Tansey MG. TNF signaling inhibition in the CNS: implications for normal brain function and neurodegenerative disease. J Neuroinflammation 2008; 5: 45.

20. Wilde GJ, Pringle AK, Sundstrom LE, Mann DA, lannotti F. Attenuation and augmentation of ischaemia-related neuronal death by tumour necrosis factor-alpha in vitro. Eur J Neurosci 2000; 12: 3863-70.

21. Pringle AK, Benham CD, Sim L, Kennedy J, Iannotti F, Sundstrom LE. Selective N-type calcium channel an- 
tagonist omega conotoxin MVIIA is neuroprotective against hypoxic neurodegeneration in organotypic hippocampal-slice cultures. Stroke 1996; 27: 2124-30.

22. Stoppini L, Buchs PA, Muller D. A simple method for organotypic cultures of nervous tissue. J Neurosci Methods 1991; 37: 173-82.

23. Paxinos $\mathrm{G}$. The mouse brain in stereotaxic coordinates. Academic Press Waltham, MA, USA 2004.

24. De Luca MT, Meringolo M, Spagnolo PA, Badiani A. The role of setting for ketamine abuse: clinical and preclinical evidence. Rev Neurosci 2012; 23: 769-80.

25. Leonoudakis D, Zhao P, Beattie EC. Rapid tumor necrosis factor alpha-induced exocytosis of glutamate receptor 2-lacking AMPA receptors to extrasynaptic plasma membrane potentiates excitotoxicity. J Neurosci 2008; 28: 2119-30. 